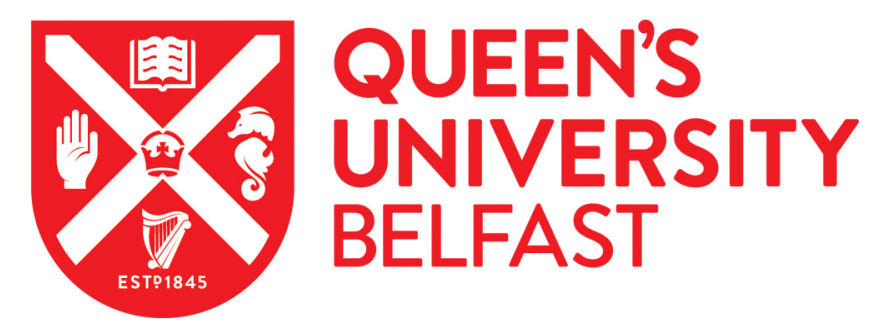

\title{
The effect of a negatively chirped laser pulse on the evolution of bubble structure in nonlinear bubble regime
}

Vosoughian, H., Riazi, Z., Afarideh, H., \& Sarri, G. (2016). The effect of a negatively chirped laser pulse on the evolution of bubble structure in nonlinear bubble regime. Physics of Plasmas, 23(12), [123113].

https://doi.org/10.1063/1.4972138

Published in:

Physics of Plasmas

Document Version:

Publisher's PDF, also known as Version of record

Queen's University Belfast - Research Portal:

Link to publication record in Queen's University Belfast Research Portal

Publisher rights

Copyright AlP 2016.

This work is made available online in accordance with the publisher's policies. Please refer to any applicable terms of use of the publisher.

\section{General rights}

Copyright for the publications made accessible via the Queen's University Belfast Research Portal is retained by the author(s) and / or other copyright owners and it is a condition of accessing these publications that users recognise and abide by the legal requirements associated with these rights.

Take down policy

The Research Portal is Queen's institutional repository that provides access to Queen's research output. Every effort has been made to ensure that content in the Research Portal does not infringe any person's rights, or applicable UK laws. If you discover content in the Research Portal that you believe breaches copyright or violates any law, please contact openaccess@qub.ac.uk. 


\section{The effect of a negatively chirped laser pulse on the evolution of bubble structure in nonlinear bubble regime}

H. Vosoughian, Z. Riazi, H. Afarideh, and G. Sarri

Citation: Physics of Plasmas 23, 123113 (2016); doi: 10.1063/1.4972138

View online: http://dx.doi.org/10.1063/1.4972138

View Table of Contents: http://aip.scitation.org/toc/php/23/12

Published by the American Institute of Physics

\section{Articles you may be interested in}

Multistage ion acceleration in the interaction of intense short laser pulse with ultrathin target

Physics of Plasmas 23, 123108123108 (2016); 10.1063/1.4971234

Effects of the precursor electron bunch on quasi-phase matched direct laser acceleration

Physics of Plasmas 23, 123110123110 (2016); 10.1063/1.4971445

Target material dependence of positron generation from high intensity laser-matter interactions

Physics of Plasmas 23, 123109123109 (2016); 10.1063/1.4971235

The sources of super-high energetic electron at relativistic circularly-polarized laser-solid interactions in the presence of large scale pre-plasmas

Physics of Plasmas 23, 123116123116 (2016); 10.1063/1.4972539

Terahertz radiation generation and shape control by interaction of array Gaussian laser beams with plasma Physics of Plasmas 23, 123105123105 (2016); 10.1063/1.4968836

Plasma-based polarization modulator for high-intensity lasers

Physics of Plasmas 23, 123107123107 (2016); 10.1063/1.4971232

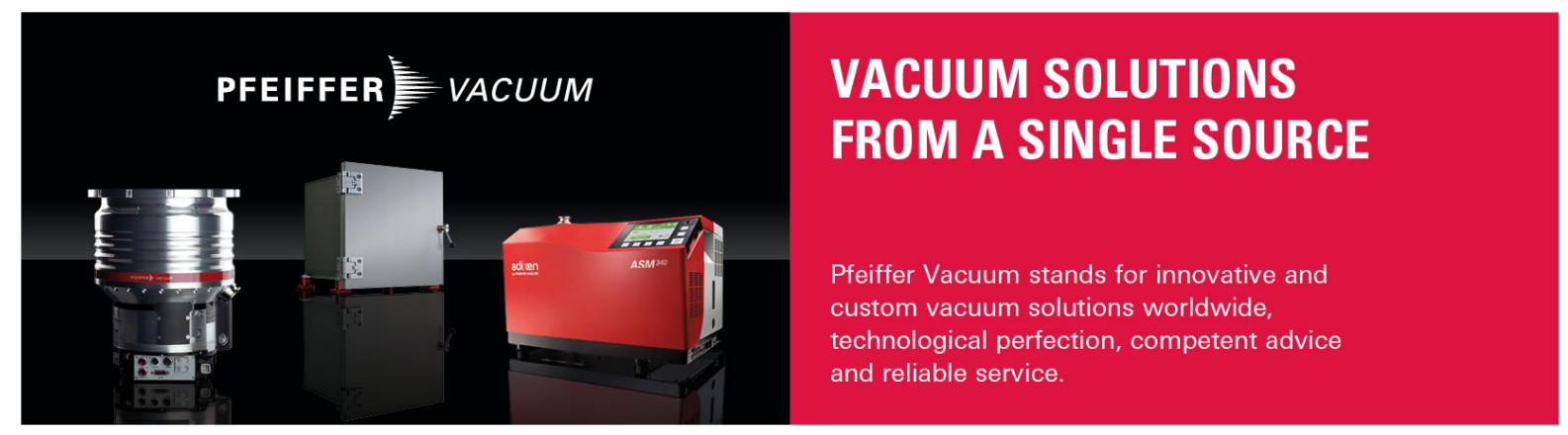




\title{
The effect of a negatively chirped laser pulse on the evolution of bubble structure in nonlinear bubble regime
}

\author{
H. Vosoughian, ${ }^{1,2,3, a)}$ Z. Riazi, ${ }^{4}$ H. Afarideh, ${ }^{2}$ and G. Sarri ${ }^{3}$ \\ ${ }^{1}$ Laser and Optic Institute, Nuclear Science and Technology Research Institute, Tehran, Iran \\ ${ }^{2}$ Department of Energy Engineering and Physics, Amirkabir University of Technology, P.O. Box 15875-4413, \\ Tehran, Iran \\ ${ }^{3}$ School of Mathematics and Physics, The Queen's University of Belfast, BT7 1NN Belfast, United Kingdom \\ ${ }^{4}$ Physics and Accelerator School, Nuclear Science and Technology Research Institute, Tehran, Iran
}

(Received 1 August 2016; accepted 28 November 2016; published online 19 December 2016)

\begin{abstract}
In the nonlinear bubble regime, due to localized depletion at the front of the pulse during its propagation through the plasma, the phase shift between carrier waves and pulse envelope plays an important role in plasma response. The Carrier-Envelope Phase (CEP) breaks down the symmetric transverse ponderomotive force of the laser pulse that makes the bubble structure unstable. Our studies using a series of two-dimensional particle-in-cell simulations show that the utilization of a negatively chirped laser pulse is more effective in controlling the pulse depletion rate, and consequently, the effect of the CEP in the bubble regime. The results indicate that the pulse depletion rate diminishes during the propagation of the pulse in plasma that leads to postponing the effect of Carrier-Envelope Phase (CEP) in plasma response, and therefore, maintaining the stability of the bubble shape for a longer time than the un-chirped laser pulse. As a result, a localized electron bunch with higher maximum energy is produced during the acceleration process. Published by AIP Publishing. [http://dx.doi.org/10.1063/1.4972138]
\end{abstract}

\section{INTRODUCTION}

Since the generation of high power femtosecond laser pulses, numerous investigations have been devoted to the study of the interaction of high-intensity lasers with plasma. One application of the laser-plasma interaction is the generation of the high-energy electron beams in which the electrons with energy up to $4.2 \mathrm{GeV}, 6 \mathrm{pC}$ charge, and $0.3 \mathrm{mrad}$ divergence have been produced from a 9-cm-long capillary discharge waveguide. ${ }^{1-12}$ The propagation of an intense laser pulse in an under-dense plasma induces a plasma wake that is suitable for the acceleration of the electrons to relativistic energies. ${ }^{13-16}$ The electrons with sufficient initial kinetic energy can ride the plasma wake, staying in phase with the longitudinal electric field inside the wake and gaining energy. Because of the small structure of the wakefield, i.e., its micron-scale longitudinal and transverse size, the bunches of a duration less than one plasma period have to be injected into the laser wakefield in order to get high-quality electron bunches. However, the effective acceleration mechanism occurs when the ambient plasma electrons contribute to the injection process (self-injection). The self-injection process is explained by the two physical mechanisms: wavebreaking (the longitudinal self-injection) ${ }^{17,18}$ and the bubble regime (transverse self-injection). ${ }^{19-21}$ The bubble regime is a multi-dimensional effect, where the symmetric transverse ponderomotive force of the laser expels the electrons radially from the propagation axis and creates a spherical region (bubble) devoid of the plasma electrons behind the laser. This bubble moves with a velocity equal to the group velocity of the laser pulse in the plasma. An intense charge

\footnotetext{
a)h.vosoughian@qub.ac.uk
}

separation electric field inside the moving bubble can capture the electrons at the base of the bubble and accelerate them with a narrow energy spread. The quality of the accelerated electrons is mainly determined by the evolution of the bubble structure. Recently, there have been several theoretical ${ }^{22,23}$ and experimental ${ }^{24}$ investigations to study the influence of the bubble evolution on the wakefield structure, and hence, on the quality of the electrons beam. It has been shown that, in the fully relativistic regime, in which the normalized vector potential of laser $a_{0}=e E_{0} / m_{e} c \omega_{0}$ approaches 1 (where $E_{0}$ and $\omega_{0}$ are the peak electric field and the frequency of the laser), the interaction of the laser with the under-dense plasma can lead to several nonlinear processes such as laser self-steepening. ${ }^{25}$ In laser self-steepening, the peak region of the pulse with high-intensity moves faster than those with low intensity at the pulse head. This leads to optical shock formation and then a nonlinear wake is excited. As a result, the plasma wavelength elongates and uninterrupted selfinjection follows. ${ }^{26-28}$ The laser pulse is depleted due to the excitation of a plasma wave. For a situation where the laser is ultra-intense $\left(a_{0} \gg 1\right)$, the pump deletion occurs at a narrow region in front of the pulse corresponding to a few cycles of the laser front. ${ }^{29}$ A consequence of the localized pulse depletion is the induction of the transverse oscillations in the bubble sheath leading to multi-injection, a large energy spread, and collective transverse oscillation of the electron beams. ${ }^{30}$ Therefore, it is important to control these instabilities to achieve high quality electron beams. In this work, the interaction of a negatively chirped laser pulse with the under-dense plasma is studied via $2 \mathrm{D}$ particle-in-cell simulations. The aim of this work is to propose a new approach to suppress the induced transverse oscillations in the bubble structure at ultra-relativistic regimes $\left(a_{0} \approx 10\right)$ 
using the frequency chirped laser pulse. Based on this technique, a considerable difference in pulse depletion process is observed for negatively chirped laser pulses than the unchirped one. This difference can be used to tune the bubble structure, and hence, the quality of the injected bunch in the nonlinear bubble regime.

\section{THEORY AND SIMULATIONS PARAMETERS}

The interaction of the short-pulse and the ultra-high intense lasers $\left(\mathrm{I}>10^{19} \mathrm{~W} / \mathrm{cm}\right)$ with the undersense plasma presents different features compared to moderately high intensities $\left(\mathrm{I}<10^{18} \mathrm{~W} / \mathrm{cm}\right)$. At moderately high intensity, the nonlinear instabilities such as relativistic self-focusing, ${ }^{31}$ relativistic self-phase modulation, ${ }^{32}$ Raman backward and forward scattering, ${ }^{33}$ and envelope self-modulation, ${ }^{34}$ cause the laser pulse to be absorbed and scattered in a distance of the order of a Rayleigh length. At ultra-high intensities, due to relativistic effects, ${ }^{35-38}$ the growth rate decreases for the above mentioned instabilities. This leads to the depletion of only the leading edge of the pulse, and the main body of the pulse undergoes little modification. Therefore, the laser pulse eventually evolves to a state in which the leading edge becomes a step function. The phase and the group velocity of the laser in a dispersive medium, such as plasma, are not equal. Therefore, propagation of a laser pulse through plasma induces a phase shift between the carrier wave and the envelope, known as the carrier-envelope phase (CEP). The time variation of the electric field for extremely short laser pulses of few-oscillations depends on the carrierenvelope phase. The localized depletion of a laser pulse at ultra-high intensities causes the varying electric field of the laser at the front of the depleted pulse to act like a few cycle pulse $\mathrm{s}^{39,40}$ in which the asymmetric transverse ponderomotive force leads to the occurrence of the transverse oscillations in the bubble structure. ${ }^{41}$ To stabilize the bubble shape, the depletion of the pulse leading edge must be mitigated in order to avoid the effect of the carrier phase shift on the transverse ponderomotive force. Since the depletion velocity of pulse, i.e., $v_{\text {depl }}=c \omega_{p}^{2} / \omega\left(\xi_{\text {depl }}\right)^{2},{ }^{29}$ depends on the laser frequency, i.e., $\omega\left(\xi_{\text {depl }}\right)$, it is possible to postpone the pulse depletion rate by locating the higher frequency at the front of the pulse. Here, $\xi_{\text {depl }}$ is the position of the localized depletion within the laser in dimensionless retarded time, $\xi$ the coordinates and $\omega_{p}$ is the plasma frequency. By this technique, we would be able to significantly suppress the transverse cavity oscillations when the pulse propagates through the plasma. In order to demonstrate the proposed scheme, the 2D PIC simulations have been carried out by EPOCH code $^{42}$ to show the effect of pulse chirping on the evolution of the bubble structure. In these simulations, the total simulation box is $200 \mu \mathrm{m}(x) \times 70 \mu \mathrm{m}(y)$ which corresponds to a window with $2000 \times 900$ cells and $6 \times 10^{6}$ particles. The under-dense hydrogen plasma $\left(T_{e}=0\right)$ is initially located in the region of $30 \mu \mathrm{m}<x<175 \mu \mathrm{m}, \quad-30 \mu \mathrm{m}<y<30 \mu \mathrm{m}$ with the density of $n_{e}=0.02 n_{c}$, where $n_{c}=1.1 \times 10^{21} \mathrm{~cm}^{-3}$ is the critical density for the laser pulse with a wave length of $\lambda_{L}=1 \mu \mathrm{m}$. The electric field of the chirped laser pulse that propagates along the $\mathrm{x}$-axis can be expressed as ${ }^{43}$

$$
E_{L y}(y, \xi)=E_{0} \exp \left(-y^{2} / \sigma_{y}^{2}\right) \exp \left(-\xi^{2} / \sigma_{x}^{2}\right) \cos (\omega(\xi) \xi) .
$$

Here, the electric field amplitude of the laser pulse is defined as $E_{0}$, which is a constant value. The local frequency of the linearly chirped pulse, the longitudinal laser length, and the laser beam waist are denoted by $\omega(\xi)=(1-b \xi), \sigma_{x}$, and $\sigma_{y}$, respectively. Here, $b<0$ denotes the dimensionless chirp parameter for the negatively chirped pulse and $\xi=\frac{(x-c t) \omega_{0}}{c}$ is the dimensionless retarded time in which $c, m_{e}, \omega_{0}$, and $e$ are the speed of light in vacuum, the plasma electron mass, the local frequency in the center of the pulse, and the absolute charge of the plasma electron, respectively. Also, $x, t$, and $c$ represent the spatial coordinate, temporal coordinate, and the speed of light, respectively. In this work, the laser beam waist, the longitudinal laser length, and the normalized incident laser amplitude are set equal to $\sigma_{y}=8 \mu \mathrm{m}, \sigma_{x}=10 \mu \mathrm{m}$, and $a_{0}=\frac{e E_{0}}{m_{e} c \omega_{0}}=10$, respectively.

\section{RESULTS AND DISCUSSION}

The bubble structure at the three different instances during the propagation of the laser pulse through the underdense plasma is shown in Fig. 1. Here, the un-chirped laser pulse with a normalized amplitude of $a_{0}=10$, a longitudinal size of $\sigma_{x}=10 \mu \mathrm{m}$, and a spot size of $\sigma_{y}=8 \mu \mathrm{m}$ enters the simulation box from the left boundary.

As it is clear from Fig. 1(a), at the initial stage of the bubble regime, i.e., at $\mathrm{t}=300 \mathrm{fs}$, the symmetric bubble structure is established, whereas, at the later times, the instabilities appear in the surrounding sheath of the bubble as seen

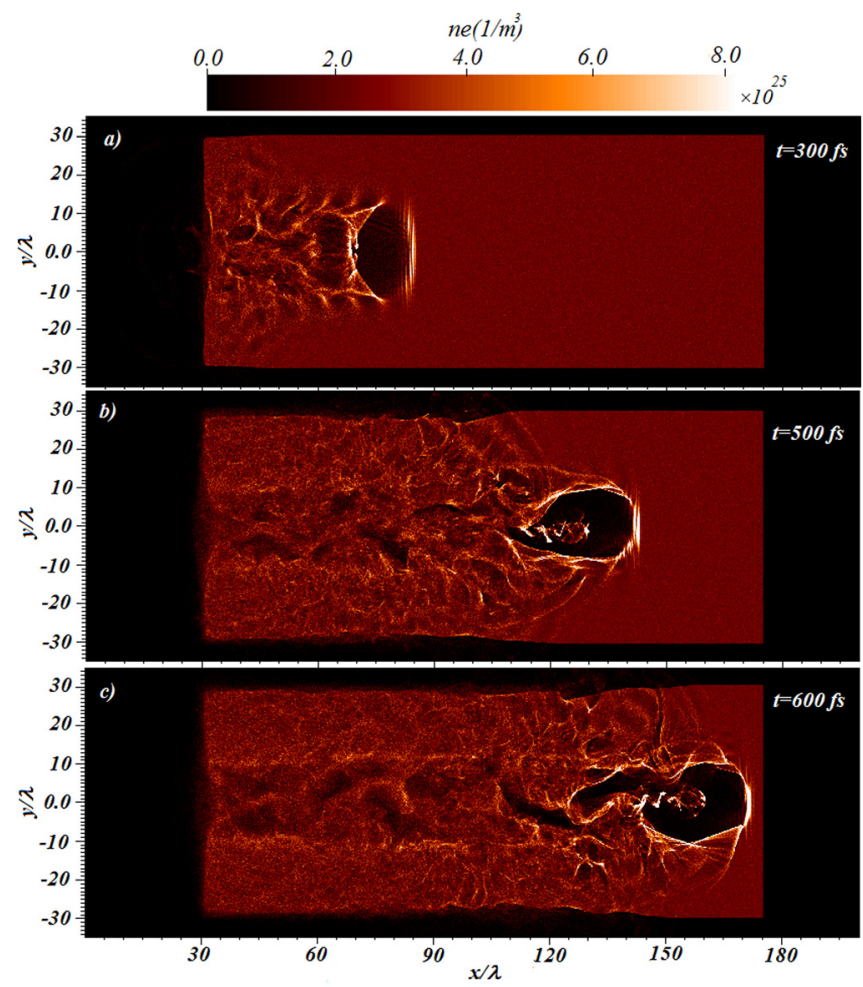

FIG. 1. The evolution of bubble structure when the un-chirped laser pulse propagates through the under-dense plasma $\left(n_{e}=0.02 n_{c}\right)$ at the times of: (a) $\mathrm{t}=300 \mathrm{fs}$, (b) $\mathrm{t}=500 \mathrm{fs}$, and (c) $\mathrm{t}=600 \mathrm{fs}$. The laser pulse with the normalized amplitude of $a_{0}=10$, the longitudinal size of $\sigma_{x}=10 \mu \mathrm{m}$, and the spot size of $\sigma_{y}=8 \mu \mathrm{m}$ enters the simulation box from the left boundary. 

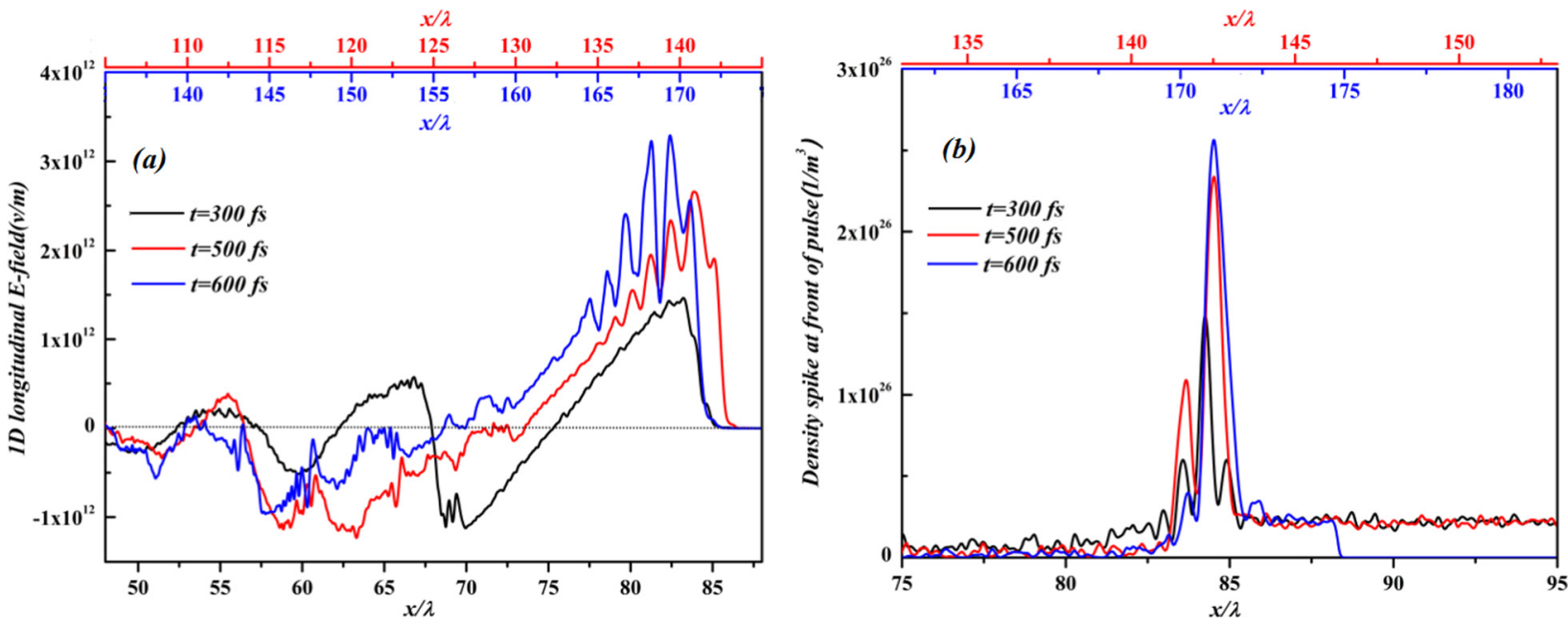

FIG. 2. (a) The excited wake-field behind the un-chirped pulse. (b) The excited density spike at the pulse head of the un-chirped laser pulse when it propagates through the under-dense plasma $\left(n_{e}=0.02 n_{c}\right)$ at the times of $\mathrm{t}=300 \mathrm{fs}, \mathrm{t}=500 \mathrm{fs}$, and $\mathrm{t}=600 \mathrm{fs}$.

in Figs. 1(b) and 1(c). When the ultra-intense laser pulse interacts with the plasma, a density spike is produced by the ponderomotive force of the front of the laser pulse and subsequently a large plasma wake is excited behind the laser. The longitudinal electric field, the plasma density at the front of the pulse, and the transverse electric field of laser are plotted in Figs. 2(a), 2(b), and 3, respectively, for $\mathrm{t}=300 \mathrm{fs}$, $\mathrm{t}=500 \mathrm{fs}$, and $\mathrm{t}=600 \mathrm{fs}$.

As shown in Figs. 2(a) and 2(b), the amplitude of wake and also the density spike continuously enhances during the time. As a result, the laser energy absorption by the electrons in the spike or pulse depletion will increase too. Viewed from a photon description, the photons localized within the density spike lose energy as they excite the wake, and therefore, their wavelength increases leading to frequency red shifting at the front of the pulse [Fig. 3].

In this situation, the front of the pulse is approximately converted to a one-cycle profile. Once the leading edge of pulse erodes, the bubble structure becomes susceptible to transverse instabilities. In fact, considering the plasma as a

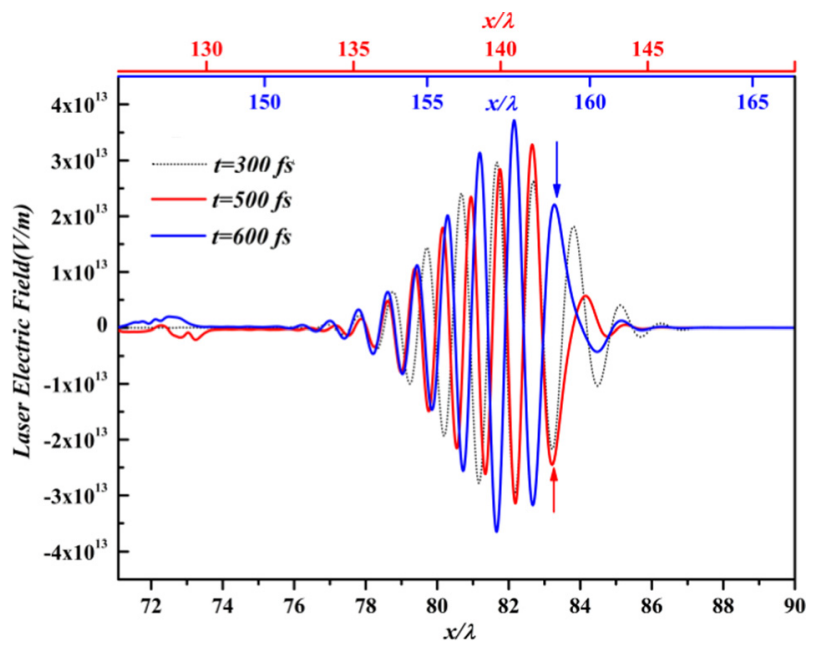

FIG. 3. The evolution of the laser's electric field of the un-chirped laser pulse during its propagation thought the under-dens plasma at the times of $\mathrm{t}=300 \mathrm{fs}, \mathrm{t}=500 \mathrm{fs}$, and $\mathrm{t}=600 \mathrm{fs}$. dispersive medium, due to the induced phase shift between the carrier waves and the pulse envelope, the role of the time average of the laser field at the front of the depleted pulse becomes dominant on the intensity envelope of the pulse in laserplasma interactions. Therefore, changing the field direction at pulse front, as depicted by the red and blue arrows in Fig. 3, causes the electrons to be asymmetrically expelled from the laser propagation axis leading to transverse oscillations in the bubble structure. In order to control the evolution of the bubble structure, we benefit from the negatively chirped laser pulse to reduce the pulse depletion rate at the pulse's leading edge. The interaction of a negatively chirped laser pulse with an underdense plasma $\left(n_{e}=0.02 n_{c}\right)$ is shown in Fig. 4.

As can be seen for the negatively chirped pulse, the symmetric structure of the moving bubble remains stable with time [Figs. 4(a)-4(c)], whereas as shown in Fig. 1(b), the cavity oscillations in the surrounding sheath of the bubble for the un-chirped pulse occur in an earlier time of interaction. The longitudinal electric field and the density spike at the pulse head for the negatively chirped pulse are depicted in Figs. 5(a) and 5(b), respectively, for $\mathrm{t}=300 \mathrm{fs}, \mathrm{t}=500 \mathrm{fs}$, and $\mathrm{t}=600$ fs.

In fact, with the higher frequencies located in front of the pulse, the plasma transparency increases at the leading part of the pulse resulting in the excitation of a small density spike [Fig. 5(b)] and also a weaker wake-field compared with the un-chirped pulse [Fig. 5(a)].

As a result, the pulse depletion rate at the leading edge of the negatively chirped pulse decreases, and thus, the carrier phase shift becomes negligible in the leading edge of the pulse as depicted in Fig. 6. Therefore, the ponderomotive force of laser keeps its symmetric properties in transverse direction that causes to maintain the conditions needed for producing the stable bubble shape during the pulse propagation.

The longitudinal electric field together with the plasma density is shown in Figs. 7(a)-7(c) for negatively chirped pulse at times $t=410,510$, and $570 \mathrm{fs}$. The electrostatic fields are denoted by the blue curves and the density distributions of injected electrons are specified by the black curves. Also, the energy spectrum of the injected electrons at the 


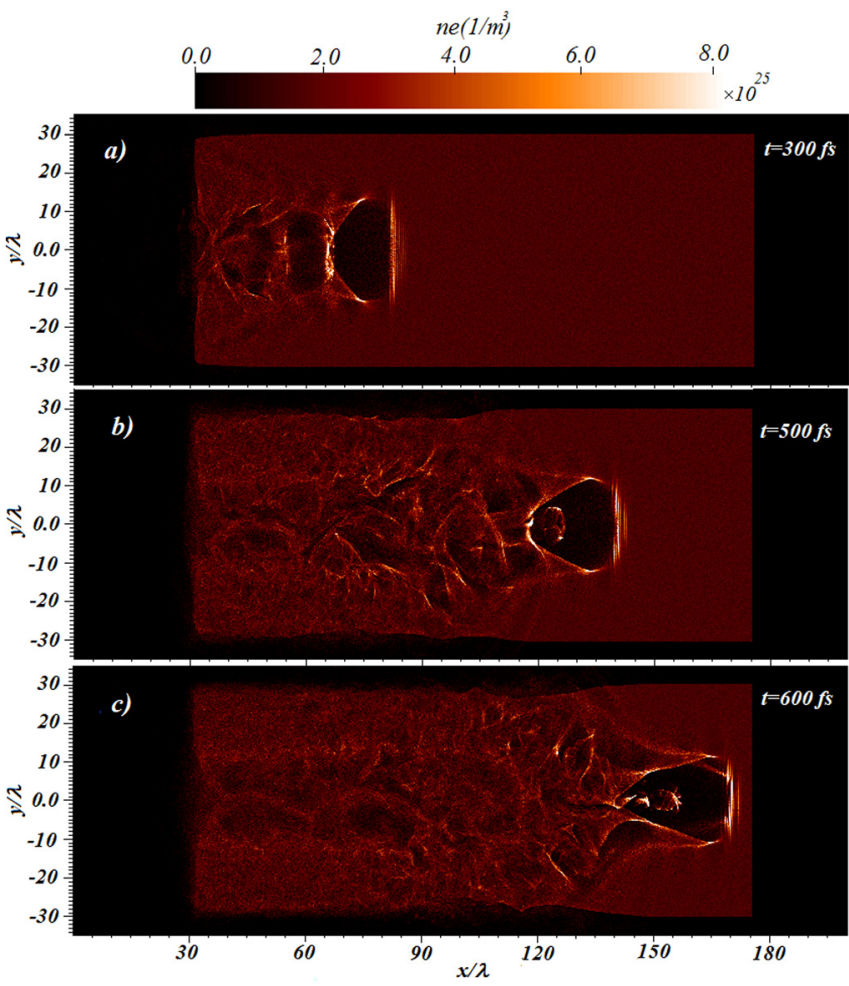

FIG. 4. The evolution of bubble structure when the negatively chirped pulse with the chirped parameter of $\mathrm{b}=-0.08$ propagates through the under-dense plasma $\left(n_{e}=0.02 n_{c}\right)$ at the times of: (a) $\mathrm{t}=300 \mathrm{fs}$, (b) $\mathrm{t}=500 \mathrm{fs}$ ), and (c) $\mathrm{t}=600 \mathrm{fs}$. The other laser parameters are th same as the un-chirped case.

corresponding time is plotted in Fig. 7(d). Figs. 8(a)-8(d) are the same as Figs. 7(a)-7(d), but for un-chirped pulse. In these figures, the electron-accelerating phase buckets (where $\mathrm{E}_{\mathrm{x}}<0$ ) and the electron-deceleration buckets (where $\mathrm{E}_{\mathrm{x}}>0$ ) of the wake wave are crosshatched by the green lines and the red lines, respectively. As seen in Figs. 7(a)-7(c), the entire injected bunch stays in phase with the accelerating region of the wakefield resulting in continuous acceleration of the electron bunch [Fig. 7(d)]. However, as can be clearly seen in Figs. 8(b)-8(d), for the un-chirped pulse, due to the evolution of the bubble base and the loading of a large number of electrons in the moving bubble, the accelerating region of the wake field at the leading part of the trapped bunch turns into a deceleration region which reduces the maximum attainable energy.

By comparing the energy spectrum of the electrons for the negatively and the un-chirped pulse [Figs. 7(d) and 8(d)], we can see that higher-energy electrons are obtained from the former and a higher number of electrons is obtained from the latter due to the multiple self-injection.

\section{CONCLUSION}

In the nonlinear bubble regime, the transverse oscillations of the cavity structure as a result of the depletion of the pulse leading edge can be controlled by applying negatively chirped laser pulses. The simulation results show that by locating the higher frequencies in front of the pulse, the pulse depletion process is postponed, and thus, the cavity structure stays stable for a longer time and a single electron bunch
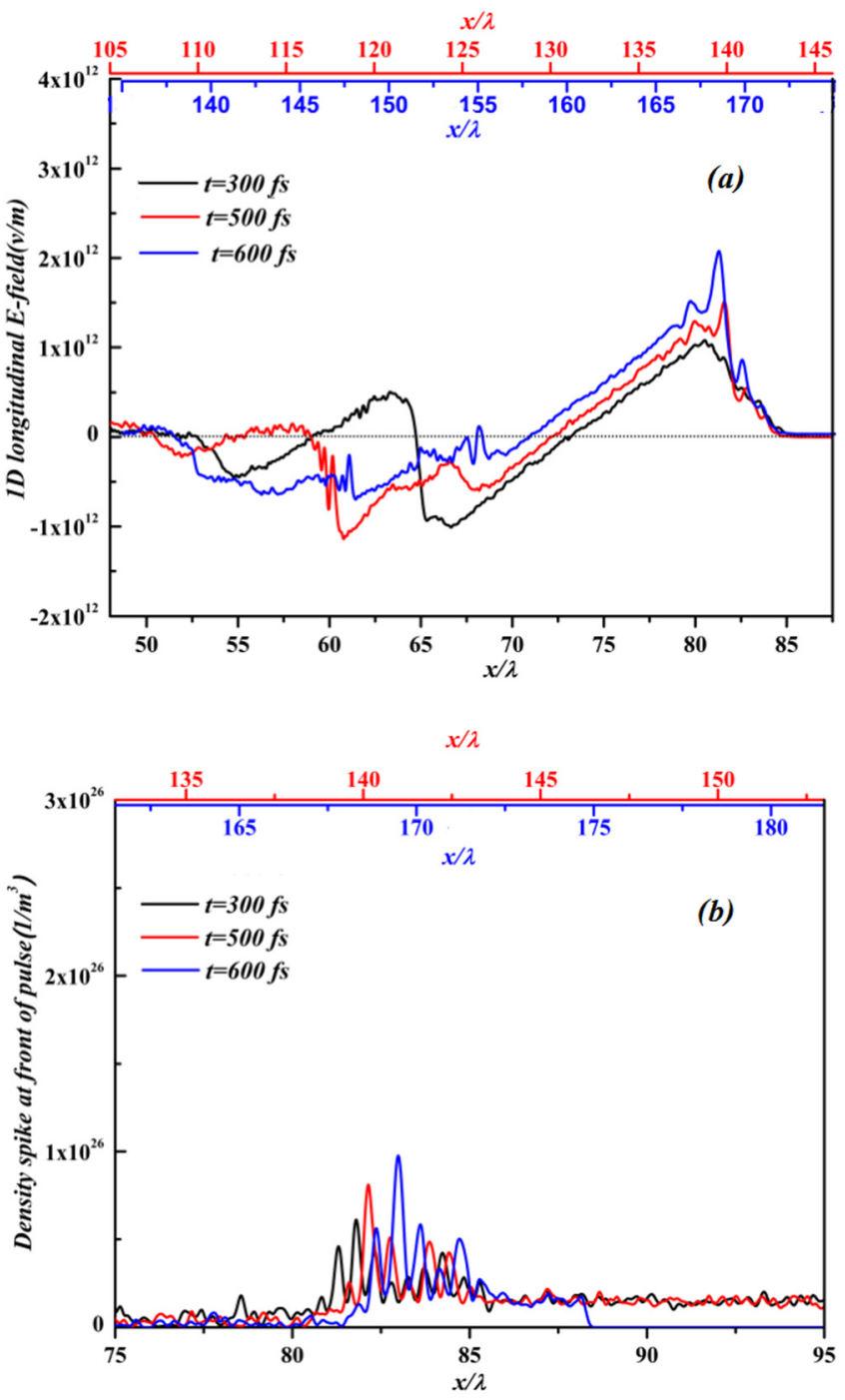

FIG. 5. (a) The excited wake-field behind the negatively chirped pulse when it propagates through the under-dense plasma $\left(n_{e}=0.02 n_{c}\right)$. (b) The electron density at the pulse head of the negatively chirped pulse at the times of $\mathrm{t}=300 \mathrm{fs}, \mathrm{t}=500 \mathrm{fs}$, and $\mathrm{t}=600 \mathrm{fs}$.

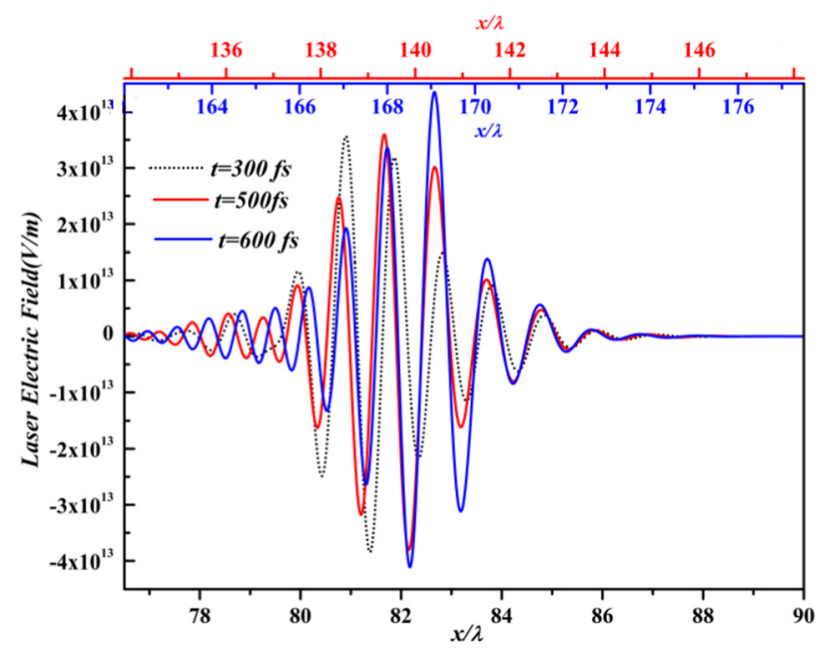

FIG. 6. The evolution of the laser's electric field of the negatively chirped pulse during its propagation thought the under-dens plasma at the times of $\mathrm{t}=300 \mathrm{fs}, \mathrm{t}=500 \mathrm{fs}$, and $\mathrm{t}=600 \mathrm{fs}$. 

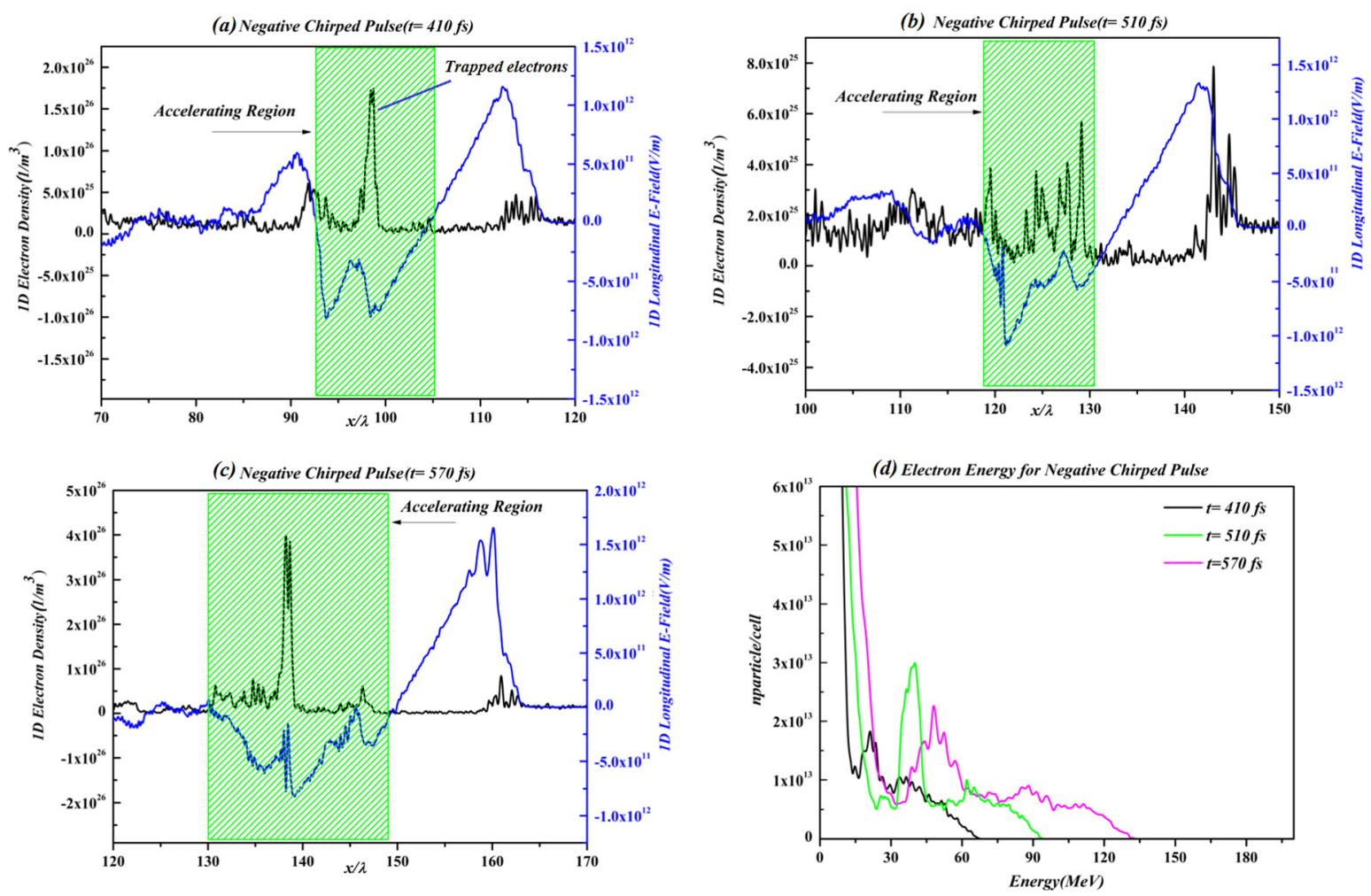

FIG. 7. The superposition of $1 \mathrm{D}$ diagrams of the wakefield structure and plasma density during the propagation of the negatively chirped pulse in plasma at the times of: (a) $410 \mathrm{fs}$, (b) $510 \mathrm{fs}$, and (c) $570 \mathrm{fs}$. The blue curves show the electrostatic field of wakefield and the black curves specify the density distribution of injected electrons. Also, the green region determines the acceleration phase of the wakefield. (d) The energy spectrum of injected electrons at the corresponding times of: $\mathrm{t}=410 \mathrm{fs}, 510 \mathrm{fs}$, and $570 \mathrm{fs}$
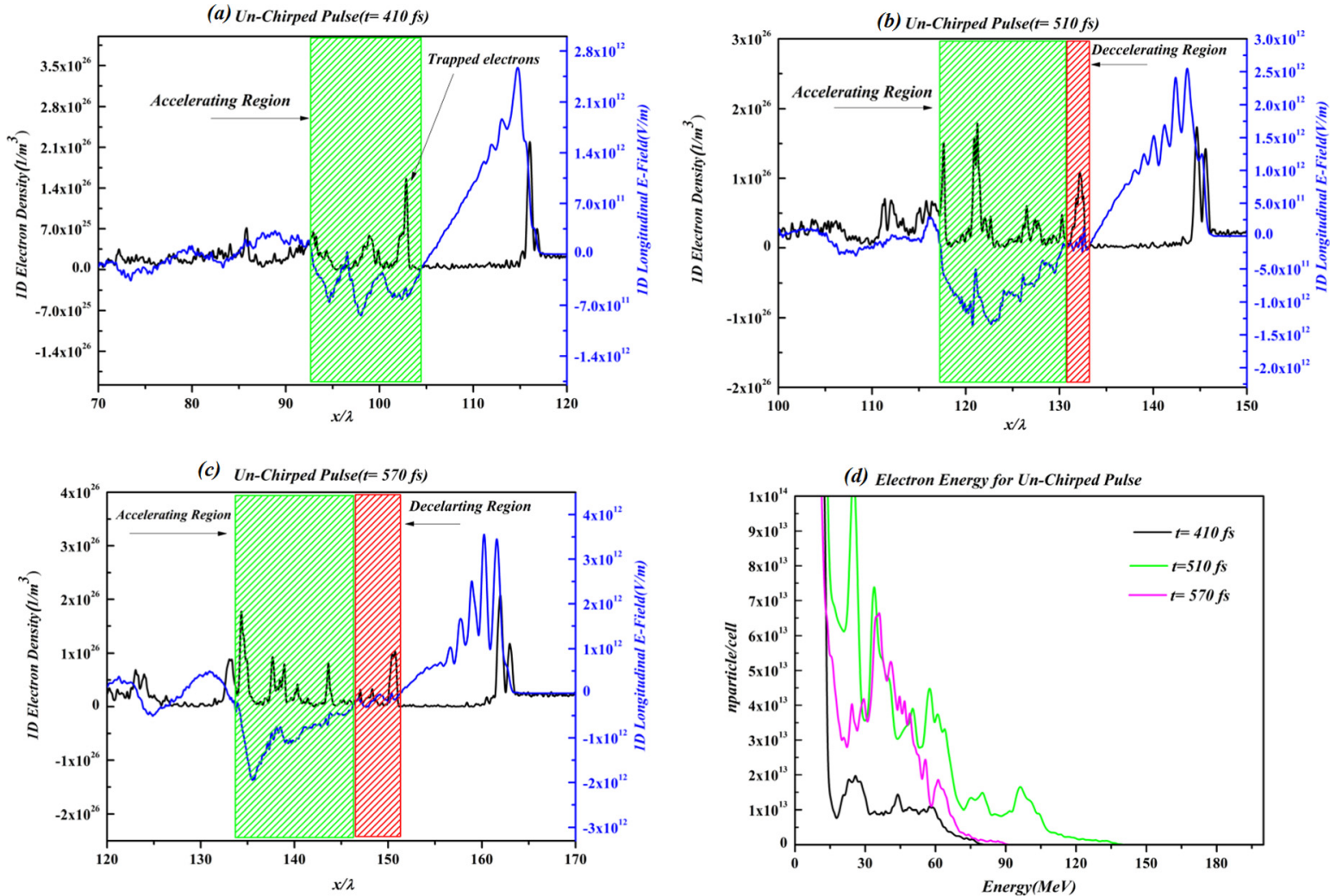

FIG. 8. The superposition of 1D diagrams of the wakefield structure and the plasma density during the propagation of the un-chirped pulse in plasma at the times of: (a) $410 \mathrm{fs}$, (b) $510 \mathrm{fs}$, and (c) $570 \mathrm{fs}$. The blue curves show the electrostatic field of the wakefield and the black curves specify the density distribution of the injected electrons. Also, the green and red regions determine the acceleration phase and deceleration phase of the wakefield, respectively. (d) The energy spectrum of the injected electrons at the corresponding times of: $t=410 \mathrm{fs}, 510 \mathrm{fs}$, and $570 \mathrm{fs}$. 
with a higher energy can be generated. In the case where the transverse instability of bubble appears in laser plasma interaction (the un-chirped pulse), the unstable bubble results in the accelerating phase of the wakefield at the leading part of the injected bunch being turned into the decelerating phase. As a consequence, the energy loss of the injected electrons causes the generation of the electron bunches with lower mean energy. Therefore, the utilization of the chirped laser pulse provides a method to control the quality of the generated bunch in the nonlinear bubble regime.

${ }^{1}$ C. G. R. Geddes, Cs. Toth, J. van Tilborg, E. Esarey, C. B. Schroeder, D. Bruhwiler, C. Nieter, J. Cary, and W. P. Leemans, Nature 431, 538 (2004).

${ }^{2}$ J. Faure, C. Rechatin, A. Norlin, A. Lifschitz, Y. Glinec, and V. Malka, Nature 444, 737 (2006)

${ }^{3}$ I. Blumenfeld, C. E. Clayton, F. J. Decker, M. J. Hogan, C. Huang, R. Ischebeck, R. Iverson, C. Joshi, T. Katsouleas, N. Kirby, W. Lu, K. A. Marsh, W. B. Mori, P. Muggli, E. Oz, R. H. Siemann, D. Walz, and M. Zhou, Nature 445, 741 (2007).

${ }^{4}$ W. Lu, M. Tzoufras, C. Joshi, F. S. Tsung, W. B. Mori, J. Vieira, R. A. Fonseca, and L. O. Silva, in Particle Accelerator Conference, PAC (2007).

${ }^{5}$ X. Y. Zhao, B. S. Xie, H. C. Wu, S. Zhang, X. R. Hong, and A. Aimidula, Phys. Plasmas 19, 033108 (2012).

${ }^{6}$ J. W. Wang, W. Yu, M. Y. Yu, H. Xu, J. J. Ju, S. X. Luan, M. Murakami, M. Zepf, and S. Rykovanov, Phys. Rev. Accel. Beams 19, 021301 (2016).

${ }^{7}$ E. Esarey, C. B. Schroeder, and W. P. Leemans, Rev. Mod. Phys. 81, 1229 (2009).

${ }^{8}$ H. Y. Su, Y. S. Huang, N. Y. Wang, X. Z. Tang, and W. Lu, Chin. Phys. Lett. 31(7), 75202 (2014).

${ }^{9}$ C. L. Yin, W. M. Wang, G. Q. Liao, M. C. Li, Y. T. Li, and J. Zhang, Acta Phys. Sin. 64(14), 144102 (2015).

${ }^{10}$ F. J. Wu, W. M. Zhou, L. Q. Shan, F. Li, D. X. Liu, Z. M. Zhang, B. Y. Li, B. Bi, B. Wu, W. W. Wang, F. Zhang, Y. Q. Gu, and B. H. Zhang, Acta Phys. Sin. 63(9), 94101 (2014).

${ }^{11}$ W. T. Li, W. T. Wang, J. S. Liu, C. Wang, Z. J. Zhang, R. Qi, C. H. Yu, R. X. Li, and Z. Z. Xu, Chin. Phys. B 24(1), 15205 (2015).

${ }^{12}$ W. P. Leemans, A. J. Gonsalves, H. S. Mao, K. Nakamura, C. Benedetti, C. B. Schroeder, D. E. Mittelberger, S. S. Bulanov, J. L. Vay, C. G. R. Geddes, and E. Esarey, Phys. Rev. Lett. 113, 245002 (2014).

${ }^{13}$ P. Sprangle, E. Esarey, J. Krall, and G. Joyce, Phys. Rev. Lett. 69, 2200 (1992).

${ }^{14}$ E. Esarey, P. Sprangle, J. Krall, A. Ting, and G. Joyce, Phys. Fluids B 5, 2690 (1993).

${ }^{15}$ L. M. Gorbunov and V. I. Kirsanov, Sov. Phys. JETP 66, 290 (1987).
${ }^{16}$ E. Esarey, P. Sprangle, J. Krall, and A. Ting, IEEE Trans. Plasma Sci. 24, 252 (1996).

${ }^{17}$ A. I. Akhiezer and R. V. Polovin, Sov. Phys. JETP 3, 696-705 (1956).

${ }^{18}$ J. M. Dawson, Phys. Rev. 113, 383 (1959).

${ }^{19}$ A. Pukhov and J. Meyer-ter Vehn, Appl. Phys. B Lasers Opt. 74, 355-361 (2002).

${ }^{20}$ A. Zhidkov, J. Koga, T. Hosokai, K. Kinoshita, and M. Uesaka, Phys. Plasmas 11, 5379 (2004)

${ }^{21}$ W. Lu, C. Huang, M. Zhou, W. B. Mori, and T. Katsouleas, Phys. Rev. Lett. 96, 165002 (2006).

${ }^{22}$ Y. Ma, M. Chen, N. A. M. Hafz, D. Z. Li, K. Huang, W. C. Yan, J. Dunn, Z. M. Sheng, and J. Zhang, Appl. Phys. Lett. 105, 161110 (2014).

${ }^{23}$ S. Lee, T. H. Lee, D. N. Gupta, H. S. Uhm, and H. Suk, Plasma Phys. Controlled Fusion 57, 075002 (2015).

${ }^{24}$ N. A. M. Hafz, S. K. Lee, T. M. Jeong, and J. Lee, Nucl. Instrum. Methods Phys. Res. A 637, S51-S53 (2011).

${ }^{25}$ F. DeMartini, T. K. Gustafson, and P. Kelley, Phys. Rev. 164, 312 (1967).

${ }^{26}$ S. Y. Kalmykov, A. Beck, S. A. Yi, V. N. Khudik, M. C. Downer, E. Lefebvre, B. A. Shadwick, and D. P. Umstadter, Phys. Plasmas 18, 056704 (2011).

${ }^{27}$ J. Faure, Y. Glinec, J. J. Santos, F. Ewald, J. P. Rousseau, S. Kiselev, A. Pukhov, T. Hosokai, and V. Malka, Phys. Rev. Lett. 95, 205003 (2005).

${ }^{28}$ S. Y. Kalmykov, A. Beck, X. Davoine, E. Lefebvre, and B. A. Shadwick, New J. Phys. 14, 033025 (2012).

${ }^{29}$ C. D. Decker, W. B. Mori, K. C. Tzeng, and T. Katsouleas, Phys. Plasmas 3, 2047 (1996).

${ }^{30}$ A. Popp, J. Vieira, J. Osterhoff, Zs. Major, R. Horlein, M. Fuchs, R. Weingartner, T. R. Rowlands-Rees, M. Marti, R. A. Fonseca, S. F. Martins, L. O. Silva, S. M. Hooker, F. Krausz, F. Gruner, and S. Karsch, Phys. Rev. Lett. 105, 215001 (2010).

${ }^{31}$ P. Sprangle, C. M. Tang, and E. Esarey, IEEE Trans. Plasma Sci. 15, 145 (1987).

${ }^{32}$ C. E. Max, J. Arons, and A. B. Langdon, Phys. Rev. Lett. 33, 209 (1974).

${ }^{33}$ W. B. Mori, C. D. Decker, T. Katsouleas, and D. E. Hinkel, Phys. Rev. Lett. 72, 1482 (1994).

${ }^{34}$ E. Esarey, J. Krall, and P. Sprangle, Phys. Rev. Lett. 72, 2887 (1994).

${ }^{35}$ S. V. Bulanov, I. N. Inovenkov, V. I. Kirsanov, N. M. Naumova, and A. S. Sakharov, Phys. Fluids B 4, 1935 (1992).

${ }^{36}$ P. Sprangle and E. Esarey, Phys. Rev. Lett. 67, 2021 (1991).

${ }^{37}$ E. Esarey and P. Sprangle, Phys. Rev. A 45, 5872 (1992).

${ }^{38}$ A. S. Sakharaov and V. I. Kirsanov, Phys. Rev. E 49, 3274 (1994).

${ }^{39}$ G. G. Paulus, F. Grasbon, H. Walther, P. Villoresi, M. Nisoli, S. Stagira, E. Priori, and S. De Silvestri, Nature. 414, 182-184 (2001).

${ }^{40}$ E. Cormier and P. Lambropoulos, Eur. Phys. J. D 2, 15-20 (1998).

${ }^{41}$ E. N. Nerush and I. Yu. Kostyukov, Phys. Rev. Lett. 103, 035001 (2009).

${ }^{42}$ See http://ccpforge.cse.rl.ac.uk/gf/project/epoch/ for "EPOCH: Extendable PIC Open Collaboration," 2011.

${ }^{43}$ S. Afhami and E. Eslami, Phys. Plasmas 21, 063108 (2014). 